\title{
Generating Normally Distributed Random Numbers by Inverting the Normal Distribution Function
}

\author{
By Friedrich Gebhardt
}

1. Introduction and Summary. Some calculations on digital computer's require a multitude of normally distributed random numbers, for instance in connection with Monte Carlo methods. Because of the great quantity, a fast way of generating them is desired, which would naturally be done at some expense of precision. Several methods are described and compared by M. E. Muller in [3]. In order to transform uniformly distributed random numbers into normally distributed ones, he proposes in [2] to approximate the inverse function of the cumulative normal distribution function by polynomials. The interval $0 \leqq t \leqq 1$ is divided into 128 parts of equal lengths, and for all of them except the first and the last one, where the inverse function becomes infinite, polynomials of first, second, and fourth degree respectively, are given, approximating the inverse distribution with a maximum error of 0.0004 . The division into 128 parts is appropriate for binary electronic computers. We give in Section 2 similar approximations for decimal computers dividing the whole interval into 100 parts for maximum errors of .0004 and .0001. Near its singularities, the inverse function can be approximated by rational functions and by an iterative method based on the semiconvergent series of the normal distribution function. This will be done in Section 3.

2. Approximation by Polynomials. Let $\Phi(x)$ be the cumulative normal distribution function and $\psi(t)$ its inverse, $0 \leqq t \leqq 1$. Because of the relation

$$
\psi(1-t)=-\psi(t)
$$

we restrict our attention to $t \leqq .5$. We want to approximate $\psi(t)$ in appropriate intervals by polynomials. In order to facilitate address modification on decimal computers, the whole range of $t$ is divided into subintervals of length $\frac{1}{100}$, the subinterval $I_{n}$ being

$I_{n}$

$$
\frac{n}{100} \leqq t<\frac{n+1}{100}, \quad n=0,1, \cdots 49 .
$$

To get a maximum error $\epsilon_{M}$ of less than 0.0004 , approximation of $\psi(t)$ by linear functions $a_{n}+b_{n} t$ is sufficient for $8 \leqq n \leqq 49$. For $n \geqq 16$, the maximum error is even less than 0.0001. The coefficients $a_{n}$ and $b_{n}$ are shown in Table 1. Quadratic functions are sufficient for $\epsilon_{M}=.0001, n \geqq 3$, and $\epsilon_{M}=.0004, n \geqq 2$, respectively, polynomials of third order for $\epsilon_{M}=.0001, n=2$, and for $\epsilon_{M}=.0004, n=1$, and a fourth order polynomial for $\epsilon_{M}=.0001, n=1$. The coefficients are listed in Table 2. The first polynomial in Table $2(n=0)$ is correct to 0.001 for $t \geqq .0020093$ only, corresponding to $\psi(t)=2.8767$; for smaller values of $t$, the error becomes fairly large, and the function value at $t=0$ is -3.283258 instead of $-\infty$. This approxi-

Received July 23, 1963. Work was done in part while the author was at the Statistische Arbeitsgruppe Prof. Dr. H. Kneser, University Tübingen. 
TABLE 1

Linear approximation of $\psi(t)$. Maximum error $\epsilon_{M}<.0004$ for all values of $n$, $\epsilon_{M}<.0001$ for $n \geqq 16$

\begin{tabular}{|c|c|c|c|c|c|}
\hline$n$ & $-a_{n}$ & $b_{n}$ & $n$ & $-a_{n}$ & $b_{n}$ \\
\hline 8 & 1.919253 & 6.4317 & 29 & 1.393892 & 2.8984 \\
\hline 9 & 1.873295 & 5.9203 & 30 & 1.380905 & 2.8551 \\
\hline 10 & 1.831555 & 5.5024 & 31 & 1.368507 & 2.8151 \\
\hline 11 & 1.793280 & 5.1541 & 32 & 1.356829 & 2.7786 \\
\hline 12 & 1.757969 & 4.8596 & 33 & 1.345743 & 2.7450 \\
\hline 13 & $\begin{array}{lll}1.725 & 181\end{array}$ & 4.6072 & 34 & $1.335 \quad 272$ & 2.7142 \\
\hline 14 & 1.694582 & 4.3885 & 35 & 1.325474 & 2.6862 \\
\hline 15 & 1.665962 & 4.1976 & 36 & 1.316259 & 2.6606 \\
\hline 16 & 1.639047 & 4.0293 & 37 & $1.307 \quad 603$ & 2.6372 \\
\hline 17 & 1.613677 & 3.8800 & 38 & 1.299624 & 2.6162 \\
\hline 18 & 1.589728 & 3.7469 & 39 & $1.292 \quad 216$ & 2.5972 \\
\hline 19 & 1.567050 & 3.6275 & 40 & 1.285417 & 2.5802 \\
\hline 20 & $1.545 \quad 557$ & 3.5200 & 41 & 1.279268 & 2.5652 \\
\hline 21 & $1.525 \quad 151$ & 3.4228 & 42 & 1.273684 & 2.5519 \\
\hline 22 & 1.505752 & 3.3346 & 43 & 1.268782 & 2.5405 \\
\hline 23 & $1.487 \quad 311$ & 3.2544 & 44 & 1.264515 & 2.5308 \\
\hline 24 & 1.469771 & 3.1813 & 45 & 1.260872 & 2.5227 \\
\hline 25 & 1.453075 & 3.1145 & 46 & 1.257974 & 2.5164 \\
\hline 26 & 1.437140 & 3.0532 & 47 & 1.255719 & 2.5116 \\
\hline 27 & 1.422024 & 2.9972 & 48 & 1.254233 & 2.5085 \\
\hline 28 & 1.407578 & 2.9456 & 49 & 1.253449 & 2.5069 \\
\hline
\end{tabular}

mation might be used, if not too many normally distributed random numbers are needed, or if a lower accuracy is sufficient near $t=0$ and $t=1$.

The linear functions are computed such, that the error is the same at both ends of the proper interval and has the same absolute value and opposite sign in its middle. The maximum absolute error is then slightly greater. The polynomials of second and higher degree, with the exception of the first one $(n=0)$, are Chebyshev approximations, which again almost minimize the maximum absolute error. The coefficients were computed first for a linear transform, $y$, of $t$, such that $y= \pm 1$ at the end points of the corresponding interval. The rounding error of each term then did not exceed $10^{-6}$. Transforming to $t$ as independent variable, the rounding error remains of the same order of magnitude, although the high order coefficients seem to provide an accuracy of four decimals only. I.e., any rounding error of the high order term is adjusted by corresponding alterations of the other coefficients to yield a total rounding error not much greater than $10^{-6}$. Much accuracy is lost if all coefficients are rounded to four decimal places. The first polynomial $(n=0)$ yields an error of absolute value 0.001 and opposite signs at six points of the interval $0.0020093 \leqq t \leqq 0.01$. The first end point is chosen so as to get as large an interval as possible with an absolute error less than 0.001 .

3. Special Methods for the End Intervals. If the accuracy of the first polynomial in Table 2 is not sufficient, then other methods must be used to invert the normal distribution function in this interval. An approximation closer than by a poly- 
TABLE 2

Approximation of $\psi(t)$ by polynomials of second, third and fourth degree

\begin{tabular}{|c|c|c|c|c|c|}
\hline$n$ & $-a_{n}$ & $b_{n}$ & $-c_{n}$ & $d_{n}$ & $-e_{n}$ \\
\hline \multicolumn{6}{|c|}{ Maximum error $<.001$ for $t \geqq .0020093$} \\
\hline 0 & 3.283258 & 268.6351 & 38726.92 & $\begin{array}{lll}3 & 186 & 023\end{array}$ & $104377 \cdot 10^{3}$ \\
\hline \multicolumn{6}{|c|}{ Maximum error $<.0004$} \\
\hline $\begin{array}{l}1 \\
2\end{array}$ & $\begin{array}{ll}2.862 & 543 \\
2.573 & 835\end{array}$ & $\begin{array}{l}73.76893 \\
31.862 \quad 55\end{array}$ & $\begin{array}{r}2360.586 \\
292.280\end{array}$ & 34721.3 & \\
\hline \multicolumn{6}{|c|}{ Maximum error $<.0001$} \\
\hline $\begin{array}{r}1 \\
2 \\
3 \\
4 \\
5 \\
6 \\
7 \\
8 \\
9 \\
10 \\
11 \\
12 \\
13 \\
14 \\
15\end{array}$ & $\begin{array}{ll}2.940 & 578 \\
2.686 & 913 \\
2.454 & 298 \\
2.363 & 419 \\
2.289 & 468 \\
2.226 & 823 \\
2.172 & 275 \\
2.123 & 837 \\
2.080 & 175 \\
2.040 & 482 \\
2.003 & 891 \\
1.970 & 004 \\
1.938 & 422 \\
1.908 & 714 \\
1.880 & 811\end{array}$ & $\begin{array}{rl}95.840 & 37 \\
45.711 & 66 \\
23.714 & 938 \\
19.115 & 067 \\
16.134 & 053 \\
14.034 & 602 \\
12.469 & 763 \\
11.255 & 154 \\
10.282 & 668 \\
9.487 & 217 \\
8.820 & 893 \\
8.255 & 343 \\
7.768 & 893 \\
7.344 & 010 \\
6.971 & 602\end{array}$ & $\begin{array}{r}4654.285 \\
851.840 \\
153.1653 \\
94.8907 \\
64.8247 \\
47.2253 \\
35.9980 \\
28.3813 \\
22.9647 \\
18.9787 \\
15.9447 \\
13.5847 \\
11.7113 \\
10.1920 \\
8.9493\end{array}$ & $\begin{array}{r}138586.9 \\
7460.8\end{array}$ & 1731093 \\
\hline
\end{tabular}

TABLE 3

Approximation of $\psi(t)$ by rational functions $R_{i}(t)=A_{i} t+B_{i}+C_{i} / t+D_{i} / t^{2}$ in the intervals $\alpha_{i} \leqq t \leqq \beta_{i}$

\begin{tabular}{|c|c|c|c|c|c|}
\hline$\alpha_{i}$ & $\beta_{i}$ & $A_{i}$ & $-B_{i}$ & $-10^{3} C_{i}$ & $10^{6} D_{i}$ \\
\hline \multicolumn{6}{|c|}{ Maximum error $<.0004$} \\
\hline 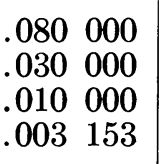 & $\begin{array}{l}.030000 \\
.010000 \\
.003153 \\
.000962\end{array}$ & $\begin{array}{rl}4.382 & 71 \\
9.462 & 73 \\
23.914 & 4 \\
66.124 & 0\end{array}$ & $\begin{array}{ll}1.535 & 054 \\
1.978 & 144 \\
2.402 & 951 \\
2.794 & 870\end{array}$ & $\begin{array}{rl}19.621 & 90 \\
6.165 & 05 \\
1.782 & 268 \\
.500 & 034\end{array}$ & $\begin{array}{r}159.3904 \\
17.4057 \\
1.60830 \\
.138558\end{array}$ \\
\hline \multicolumn{6}{|c|}{ Maximum error $<.0001$} \\
\hline $\begin{array}{ll}.010 & 000 \\
.004 & 4649 \\
.001 & 9609 \\
.000 & 8480\end{array}$ & $\begin{array}{ll}.004 & 4649 \\
.001 & 9609 \\
.000 & 8480 \\
.000 & 3624\end{array}$ & 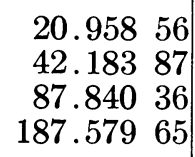 & $\begin{array}{ll}2.342 & 2273 \\
2.624 & 0660 \\
2.890 & 1189 \\
3.141 & 6999\end{array}$ & 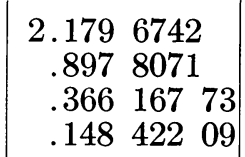 & 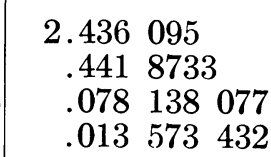 \\
\hline
\end{tabular}


nomial of fourth degree is given by the functions

$$
R_{i}(t)=A_{i} t+B_{i}+\frac{C_{i}}{t}+\frac{D_{i}}{t^{2}}, \quad \alpha_{i} \leqq t \leqq \beta_{i}
$$

which need about the same computation time on digital computers. Table 3 shows the coefficients of four such functions with a maximum error of 0.0004 in the interval $.000962 \leqq t \leqq 0.08$ and of four functions with a maximum error of 0.0001 in the interval $.0003624 \leqq t \leqq 0.01$. In the first case, the use of all four rational functions eliminates the need of quadratic and cubic polynomials as described in Section 2. This simplifies the program; however, the rational functions need about one and a half times the computation time of a quadratic polynomial.

In the remaining interval, $t<.000962, t<.0003624$ respectively, i.e. $x<-3.1016, x<-3.3800$ respectively, the approximation

$$
\begin{aligned}
\Phi(x) \approx S(x) & =\frac{\phi(x)}{T(x)}, \\
T(x) & =-x-\frac{1}{x}+\frac{2}{x^{3}}-\frac{6}{x^{5}}
\end{aligned}
$$

may be used. It is derived from the semi-convergent series for $\Phi(x) / \phi(x)$; the coefficient of $x^{-5}$, however, is altered in order to yield a smaller absolute error. Let $t$ be given, and $x, x^{*}, \Delta x$ be the solutions of

$$
\begin{aligned}
t & =\Phi(x), \\
t & =S\left(x^{*}\right), \\
x^{*} & =x+\Delta x .
\end{aligned}
$$

Then the first terms of the power expansion yield

$$
\begin{gathered}
S\left(x^{*}\right)=\Phi\left(x^{*}-\Delta x\right) \approx \Phi\left(x^{*}\right)-\Delta x \phi\left(x^{*}\right), \\
\Delta x \approx \frac{\Phi\left(x^{*}\right)}{\phi\left(x^{*}\right)}-\frac{1}{T\left(x^{*}\right)} .
\end{gathered}
$$

Numerical evaluations of this expression show that $|\Delta x|<.000067$ for $t<$ $.001(x<-3.09)$.

The solution $x^{*}$ is obtained by an iterative procedure. Let $x_{k}$ be the $k$ th approximation and

$$
\begin{aligned}
Q & =\frac{S\left(x_{k}\right)-t}{S\left(x_{k}\right)}, \\
\Delta x_{k} & =\frac{Q+Q^{2} / 2+Q^{3} / 3}{x_{k}+\frac{1}{x_{k}}} \\
x_{k+1} & =x_{k}+\Delta x_{k} .
\end{aligned}
$$

This procedure converges considerably faster than Newton's method for $S(x)$, and was derived from Newton's method applied to $\log S(x)$. Numerical calcula- 
tions showed: If $t<.001$ and $\left|\Delta x_{k}\right|<.02$, then $\left|x_{k+1}-x^{*}\right|<.00035$ and, as $\left|x_{k+1}-x^{*}\right|$ and $\left|x^{*}-x\right|$ depend on $t$ and assume their maxima at different points, $\left|x_{k+1}-x\right|<.0004$ (even $<.00037$ ). Thus, the iteration may be ended as soon as $\left|\Delta x_{k}\right|<.02$, if an accuracy of .0004 is sufficient. In the same way, if $t<.001$ and $\left|\Delta x_{k}\right|<.0035$, then $\left|x_{k+1}-x^{*}\right|<.00052,\left|x_{k+1}-x\right|<.0001$. As initial values, $x_{0}$, of the iteration process are recommended in the first case $\left(\epsilon_{M}<.0004\right) \psi(.000962)-.020=-3.122$ and in the second case $\left(\epsilon_{M}<.0001\right)$ $\psi(.0003624)-.0035=-3.3835$ in order to cover the greatest possible $t$-interval with a single iteration. In the first case, 3 iterations are needed for $t=0.0001$ and 4 for $t=0.00001$.

A program to compute $\psi(t)$ was written for the Siemens 2002 computer, using linear approximations, rational functions $R_{i}(t)$ and the iteration process, $\epsilon_{M}=$ 0.0004 . One iteration needed about the time of 40 multiplications. However, in most values of $t$, the iteration process is not involved, and the average computation time was approximately that of four and a half multiplications. This program was part of a multidimensional integration problem [1], where more than 900000 normal deviates were computed.

University of Connecticut

Storrs, Connecticut

1. Friedrich Gebhardt, "On the risk of some strategies for outlying observations," submitted to Ann. Math. Statist.

2. Mervin E. Muller, "An inverse method for the generation of random normal deviates on large-scale computers," MTAC, v. 12, 1958, p. 167-174.

3. Mervin E. Muller, "A comparison of methods for ganerating normal deviates on digital computers," J. Assoc. Comput. Mach., v. 6, 1959, p. 376-383.

\title{
Optimum Runge-Kutta Methods
}

\section{By T. E. Hull and R. L. Johnston}

\begin{abstract}
The optimum Runge-Kutta method of a particular order is the one whose truncation error is a minimum. Various measures of the size of the truncation error are considered. The optimum method is practically independent of the measure being used. Moreover, among methods of the same order which one might consider using the difference in size of the estimated error is not more than a factor of 2 or 3 . These results are confirmed in practice insofar as the choice of optimum method is concerned, but they underestimate the variation in error between different methods.
\end{abstract}

1. Introduction. For the solution of

$$
y^{\prime}=f(x, y), \quad y\left(x_{0}\right)=y_{0}
$$

the general Runge-Kutta method of order $m$ uses the formula

$$
y_{n+1}=y_{n}+\sum_{i=1}^{m} w_{i} k_{i}
$$

Received September 24, 1963, revised November 19, 1963. This research was supported in part by the Defence Research Board of Canada. 